\title{
Estimation of ionosphere state in AURORA online data analysis system
}

\author{
Yuryi Polozov*, and Nadezhda Fetisova \\ Institute of Cosmophysical Research and Radio Wave Propagation FEB RAS, 684034, Kamchatsky \\ krai, Paratunka, Russian Federation
}

\begin{abstract}
The paper presents the results of detection of ionospheric anomalies in online mode according to the ionosonde data at Paratunka station, Kamchatka peninsula (IKIR FEB RAS). The developed algorithms have been implemented in Aurora system for online geophysical data analysis (http://saoperanalysis.ikir.ru:9180/lsaoperanalysis.html). The algorithms allow us to detect sudden anomalous changes of varying intensity in the dynamics of ionospheric parameters, as well as to estimate their characteristics. The efficiency of the system and the possibility of its application in space weather forecast tasks have been shown on the examples of events occurred in 2019.
\end{abstract}

\section{Introduction}

The paper presents the results of ionospheric anomalies detection in online mode based on the ionosonde data at Paratunka station, Kamchatskiy kray (IKIR FEB RAS). To detect the anomalies, we apply a model and the algorithms for the analysis of complicated time series $[1,2,3,4]$. Ionospheric data (foF2, for example) refer to such time series. Their behavior depends on many factors such as solar activity, interplanetary magnetic field change, geomagnetic disturbances and so on $[5,6,7,8,9,10]$. Ionospheric data consists of different time oscillations: long (seasonal variations) and short-period (diurnal variations) regular changes as well as irregular disturbances of different duration and amplitude. Strong ionospheric disturbances make significant impact on the operation of radio channels, may cause disorders in the functioning of ground and satellite technical devices [7, 8, 11]. They manifest in the form of significant changes (decrease or increase) of electron concentration and are called ionosperic storms [5, 8]. Ionospheric storms have individual profiles which differ in duration, intensity and may have positive and negative phases $[2,5,6,8]$. In the recorded foF 2 ionospheric data they cause long changes of time variation and short-period local changes of different intensity and duration. Timely detection of ionospheric disturbances is an important task $[8,11]$.

Traditional methods of time series analysis determine trend components (regression, smoothing, spectral, ARIMA etc.) well and are hardly realized to detect anomalous changes in the data to which ionospheric disturbances refer $[1,8,12]$. To process the nonstationary data, we suggest using the developed Generalized Multi-Component Model (GMCM). The proposed model is aimed at modeling of foF2 ionospheric parameter time series taking into account diurnal and seasonal changes and the level of solar activity $[1,2]$. Moreover, the

*Corresponding author: up_agent@mail.ru 
model also allows us to detect anomalous changes of different duration and intensity $[1,2$, 4]. Analysis of the regular variation and anomalous features in the model is based on a complex approach including the ARIMA methods, wavelet analysis and decision rule group obtained in the result of statistic processing of measurement results.

Based on the generalized multi-component model, numerical algorithms and program tools for ionospheric parameter analysis were implemented [3, 4, 13]. With their help, it is possible to detect anomalous changes in the recorded ionospheric parameters and to estimate ionospheric disturbances in online mode. Analysis results are available in the system of online analysis on IKIR FEB RAS site http://lsaoperanalysis.ikir.ru/lsaoperanalysis.html (the system is included into Common Use Center "North-Eastern Heliogeophysical Center" CKP_558279). The system shows the changes in ionospheric data, magnetic and cosmic ray data in graphic form with different time specification $[14,15]$. Conclusions on natural time series behavior are presented in text form. On the basis of this system, ionospheric disturbances for 2019 were analyzed. The obtained results of the analysis are described in this paper.

\section{Generalized multi-component model of ionospheric parameters}

Ionospheric parameter time series may be represented in the form of a generalized multicomponent model (GMCM) [1, 2]:

$$
\mathrm{f}(\mathrm{t})=\mathrm{R}(\mathrm{t})+\mathrm{U}(\mathrm{t})+\mathrm{e}(\mathrm{t})=\sum_{\mu=\overline{1, T}} \alpha^{\mu}(\mathrm{t})+\sum_{\eta} \beta_{\mathrm{dist}}^{\eta}(\mathrm{t})+\mathrm{e}(\mathrm{t})
$$

where $\mathrm{R}(\mathrm{t})=\sum_{\mu=\overline{1, \mathrm{~T}}} \alpha^{\mu}(\mathrm{t})(\mu=\overline{1, \mathrm{~T}}$ is a component number $)$ is a regular component describing characteristic variations of ionospheric parameters and it includes the components $\alpha^{\mu}(\mathrm{t})$ determined by local factors (season, recording station, solar activity level etc.); $\mathrm{U}(\mathrm{t})=\sum_{\eta} \beta_{\mathrm{d} i s t}^{\eta}(\mathrm{t})$ is an irregular (anomalous) component describing different-scale changes of ionospheric parameters during disturbed periods (solar flare events, magnetospheric disturbances etc.); $\mathrm{e}(\mathrm{t})$ is a random component.

Applying the identification algorithm for regular component $\mathrm{R}(\mathrm{t})$ [1] of model (1), based on multi-scale wavelet decompositions (MRA) and the ARIMA models [17], R(t) can be represented in a parametric form:

$$
\mathrm{R}(\mathrm{t})=f_{-m^{r e g}}(t)+\sum_{j^{r e g}} g_{j} \text { reg }(t)=\sum_{\mu=\overline{1, \mathrm{~T}}} \sum_{\mathrm{k}=1, \mathrm{~N}_{\mathrm{j}}^{\mu} \mathrm{reg}} \mathrm{s}_{\mathrm{j}^{\mathrm{reg}}, \mathrm{k}}^{\mu} \mathrm{b}_{\mathrm{j}^{\mathrm{reg}}, \mathrm{k}}^{\mu}(\mathrm{t}),
$$

where $f_{-m^{\text {reg }}}(t)$ and $g_{j}$ reg $(t)$ are regular smoothed and detailing components of scale $m^{\text {reg }}$ $\left(j^{\text {reg }}=\overline{-1,-m^{\text {reg }}}\right) \quad$ detected on the basis of MRA;

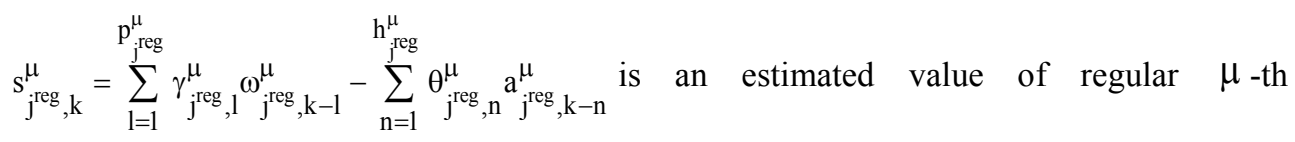
component, $\mathrm{p}_{\mathrm{j} \text { reg }}^{\mu}, \quad \gamma_{\mathrm{j} \text { reg }, 1}^{\mu}$ are the order and parameters of autoregression of $\mu$-th component, $h_{j}^{\mu}, \theta_{j}^{\mu}{ }_{j}$ reg,$n$ are the order and parameters of moving average of $\mu$-th component, $\omega_{\mathrm{j}}^{\mu}{ }_{\text {reg }}=\nabla^{\nu^{\mu}} \delta_{\mathrm{j}^{\text {reg }}, \mathrm{k}}^{\mu}, \nu^{\mu}$ is a difference order of $\mu$-th component, 
$\delta_{-\mathrm{m}^{\mathrm{reg}}, \mathrm{k}}^{1}=\mathrm{c}_{-\mathrm{m}^{\mathrm{reg}}, \mathrm{k}}, \quad c_{-m^{r e g}, k}=\left\langle f, \phi_{-m^{r e g}, k}\right\rangle$ are decomposition coefficients of MRA smoothed component, $\phi_{-\mathrm{m}^{r e g}, \mathrm{k}}(\mathrm{t})=2^{-\mathrm{m}^{r e g} / 2} \phi\left(2^{-\mathrm{m}^{r e g}} \mathrm{t}-\mathrm{k}\right)$ is a scaling function; $\delta_{\mathrm{j}^{\text {reg }}, \mathrm{k}}^{\mu}=\mathrm{d}_{\mathrm{j}}{ }_{\text {reg }, \mathrm{k}}, \mu=\overline{2, \mathrm{~T}}, \mathrm{~d}_{\mathrm{j}}{ }_{\text {reg }, \mathrm{k}}=\sum_{\mathrm{k}} \mathrm{d}_{\mathrm{j}}{ }_{\text {reg }}{ }_{\mathrm{k}} \Psi_{\mathrm{j}^{\text {reg }}, \mathrm{k}}(\mathrm{t})$ are decomposition coefficients of MRA detailing component, $T$ is the number of modeled components, $a_{j^{r e g}, k}^{\mu}$ are residual errors of the $\mu$-th component model, $\mathrm{N}_{j}^{\mu}$ reg is the length of $\mu$-th component, $\mathrm{b}_{-\mathrm{m}^{1}{ }^{\mathrm{reg}}, \mathrm{k}}=\phi_{-\mathrm{m}^{\mathrm{reg}}, \mathrm{k}}$ is a scaling function, $\mathrm{b}_{\mathrm{j}^{\mathrm{reg}}, \mathrm{k}}^{\mu}=\Psi_{\mathrm{j}^{\mathrm{reg}}, \mathrm{k}}, \mu=\overline{2, \mathrm{~T}}$ is a wavelet basis of $\mu$ -th component.

As long as the components $\beta_{\text {dist }}^{\eta}(\mathrm{t})$ of the irregular component $\mathrm{U}(\mathrm{t})$ of model (1) have local structure exposed to changes at random times, the most effective way to describe them is to apply nonlinear adaptive approximating schemes and the following ratio is true $[2,4]$ :

$$
U(t)=\sum_{\eta} \beta_{d i s t}^{\eta}(t)=\sum_{(\eta, n) \in P_{M}} d_{\eta, n} \Psi_{\eta, n}(t)+\sum_{(\eta, n) \notin P_{M}} d_{\eta, n} \Psi_{\eta, n}(t)=U_{M}(t)+e(t),(3)
$$

where $U_{M}(t)=\sum_{(\eta, n) \in P_{M}}\left\langle f, \Psi_{\eta, n}\right\rangle \Psi_{\eta, n}(t)$ is the projection of $U(t)$ on $M$ vectors, the indexes of which are contained is some set $P_{M}$; component $e(t)=\sum_{(\eta, n) \notin P_{M}}\left\langle f, \Psi_{\eta, n}\right\rangle \Psi_{\eta, n}(t)$ is a sequence of noise factor effect (it is assumed that this component is uncorrelated and additive).

In such a case, identification of component $U_{M}(t)$ may be based on the application of threshold functions $[2,4]$

$$
U_{M}(t)=\left\{\begin{array}{l}
\sum_{\eta, n} d_{\eta, n} \Psi_{\eta, n}(t), \text { if }\left|d_{\eta, n}\right| \geq T_{\eta} \\
0, \text { if }\left|d_{\eta, n}\right|<T_{\eta}
\end{array}\right.
$$

The identified model was implemented in software and is available in Internet (http://aurorasa.ikir.ru:8580, $\quad \underline{\text { http://lsaoperanalysis.ikir.ru/lsaoperanalysis.html). The }}$ scheme of software implementation of the model is illustrated in Fig. 1.

\section{Detection of ionospheric disturbances on the basis of generalized multi-component model}

Assuming that residual errors of component $\mathrm{R}(\mathrm{t})$ increase during strong ionospheric disturbances (ratio (2)), the operation of detection of intensive anomalous changes may be carried out on the basis of a conditional test $[1,4]$ :

$$
\varepsilon_{j}^{\mu} \mu^{r e g}=\sum_{q=1}^{Q_{\mu}}\left|a_{j}^{\mu}{ }^{\mu} g_{, k+q}\right|>H_{\mu, j} \text { reg, }
$$

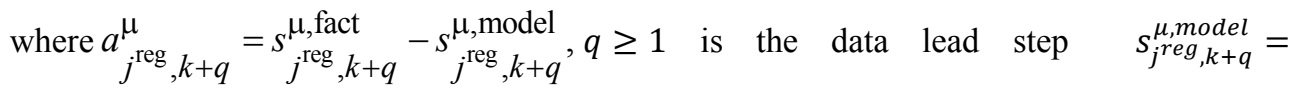

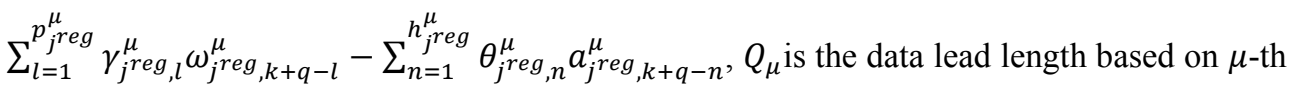
component model, $H_{\mu, j}$ reg is a threshold value of $\mu$-th component determining the presence 
of anomalous changes in $\mu$-th component $\left(H_{\mu, j}\right.$ reg was determined on the basis of model error dispersion estimate taking into account probability limits $[1,4,6])$.

Taking into account the significant non-stationarity of the modeled time series for detection of different-scale short-period anomalous changes, adaptive thresholds $T_{\eta}=T_{\eta}^{a d}$ and coefficients $\left\{d_{\eta, n}\right\}_{(\eta, m) \in P_{M}}$ were introduced in the paper in ratio (4) and set to be equal $[2,4]$

$$
d_{\eta, n}= \begin{cases}d_{\eta, n}^{+}, & \text {if }\left(d_{\eta, n}-d_{\eta, n}^{\text {med }}\right) \geq T_{\eta}^{\text {ad }} \\ d_{\eta, n}^{-}, & \text {if }\left(d_{\eta, n}-d_{\eta, n}^{\text {med }}\right) \leq-T_{\eta}^{a d}\end{cases}
$$

where $T_{\eta}^{a d}=V^{*} S t_{\eta}, S t_{\eta}=\sqrt{\frac{1}{\Phi-1} \sum_{n=1}^{\Phi}\left(d_{\eta, n}-\overline{d_{\eta, n}}\right)^{2}}, \overline{d_{\eta, n}}$ and $d_{\eta, n}^{\text {med }}$ are average value and the median, respectively. They are calculated in the framework of a moving time window of length $\Phi$ taking into account the diurnal variation.

As long as the amplitude of wavelet coefficient $\left|d_{\eta, n}\right|$ characterizes the amplitude of an anomalous feature on scale $\eta$ (see [16]), it is logical to determine it as a measure of anomaly intensity on scale $\eta$. Anomaly intensity at a time instant $t=n$ is determined as

$$
I_{n}=\sum_{\eta}\left|d_{\eta, n}\right| \text {. }
$$

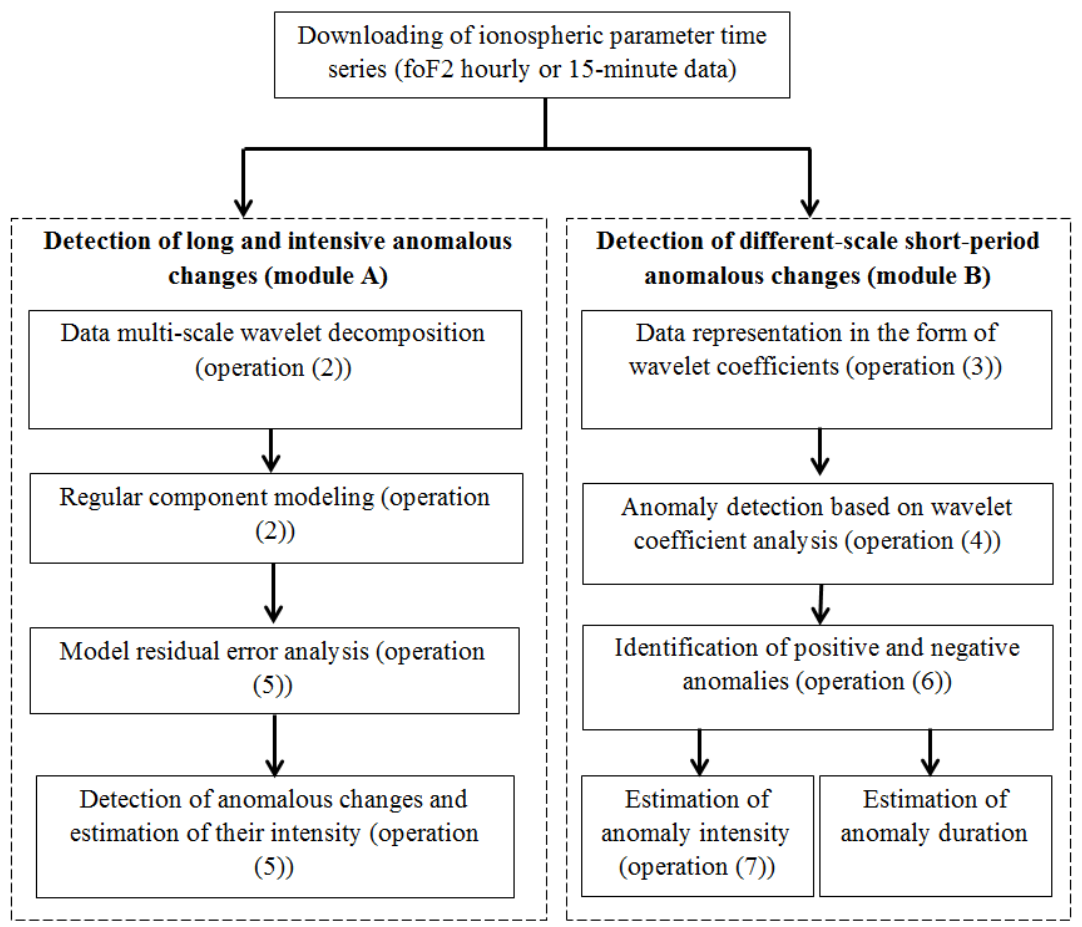

Fig. 1. Scheme of GMCM software implementation. 


\section{Results of detection of ionospheric anomalies in online mode}

Fig. 2-5 shows the analysis results of ionosphere state over Kamchakta (based on the data of ionospheric critical frequency foF2 at Paratunka station) during geomagnetic disturbances in 2019. To analyze solar and geomagnetic activity, Fig. 3, 5 show solar wind velocity and Earth magnetic field indexes (graphs were obtained by IKIR FEB RAS online analysis system http://lsaoperanalysis.ikir.ru:9180/lsaoperanalysis.html). Processing of foF2 ionospheric data was based on the developed methods and algorithms implemented in online mode in Aurora software system (http://saoperanalysis.ikir.ru:9180/lsaoperanalysis.html). As the data come to the system, the processing results are displayed as graphs and in text form. The history of processing results since 2018 is also available in the system archive.

Fig. 2, 3 shows the processing results for foF2 ionospheric data during a geomagnetic storm on January 31 - February 1, 2019. According to the data of space weather site (http://ipg.geospace.ru), inhomogeneous accelerated flux from the western part of a coronal whole arrived on January 31 at about 15.00 UT, solar wind velocity increased to $550 \mathrm{~km} / \mathrm{s}$, IMF southern component fluctuations grew to $\mathrm{Bz}= \pm 16 \mathrm{nT}$ (Fig. 3a, b). Then, on February 1, solar wind velocity kept growing and reached the maximum value of $640 \mathrm{~km} / \mathrm{s}$, IMF southern component fluctuations were $\mathrm{Bz}= \pm 6 \mathrm{nT}$ (Fig. $3 \mathrm{a}, \mathrm{b}$ ). Up to the end of the period under analysis (February 4, 2019) solar wind velocity was within 450-500 km/sec. Fig. $2 \mathrm{~b}$ and $3 \mathrm{e}$ show ionospheric anomalies detected on the bases of operation (6). Electron concentration increase (positive anomaly) observed during the disturbed period is marked by red, the decrease (negative anomaly) is marked by blue. The intensity of the detected anomalies is illustrated in Fig. 2c and $3 f$ (operation (7)). The positive anomaly intensity reached the maximum values at the beginning of the day on February 1 (UT). The results of ionospheric data modeling (ratio (5), fiductial probability is $70 \%$, Fig. 2 d,e) show that during the disturbed period the ionospheric process dynamics changed, long (about $32 \mathrm{~h}$ ) changes in foF2 time variation (2.4 of standard deviation, Fig. 2d) and short-period oscillating changes (1.6 of standard deviation, Fig 2e) are observed. As it is clear from the graphs (Fig. 3 e,f), a positive anomaly of weak intensity with the maximum at about 13.00 (UT) was detected in the ionosphere on January 31 several hours before the sudden oscillations in the data of solar wind velocity, IMFBz and the Earth magnetic field (Fig. 3a, b, c). Comparison of foF2 median values and the initial time series (Fig. 2a) show shortterm increase of electron concentration during the detection of the positive anomaly of weak intensity. A similar result was obtained for the event described below. A positive anomaly of weak intensity was observed in the ionosphere before a magnetic storm (Fig. 4, 5). 
foF2 data

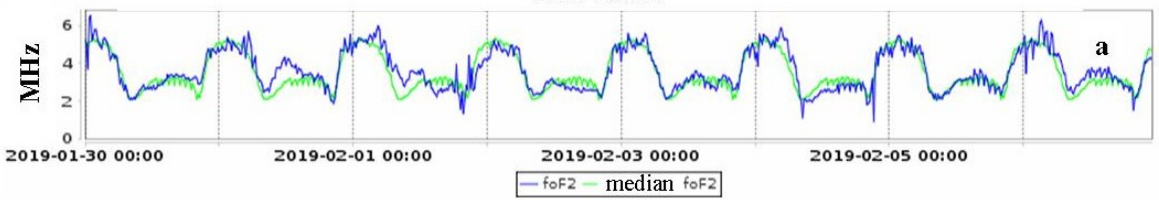

Disturbed periods

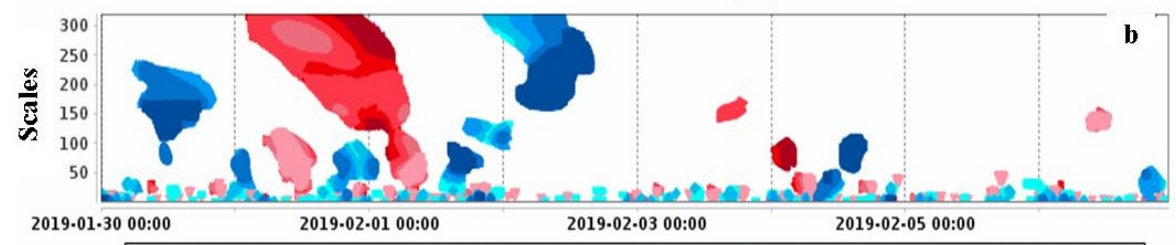

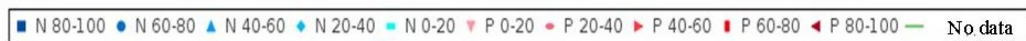

Intensity of disturbed periods

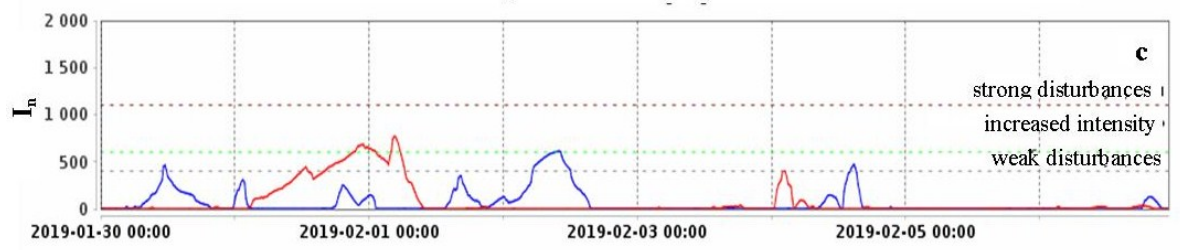

Errors of smoothed component modeling

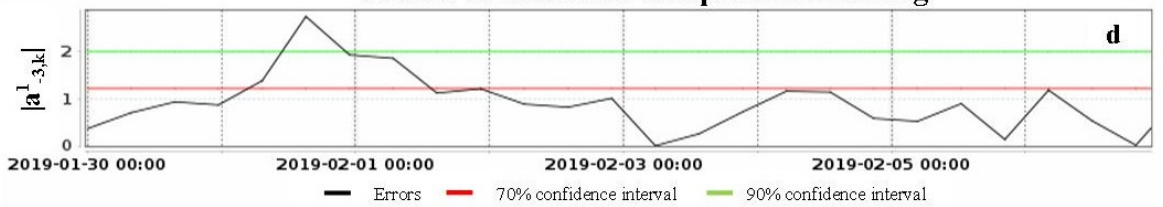

Errors of detailing component modeling

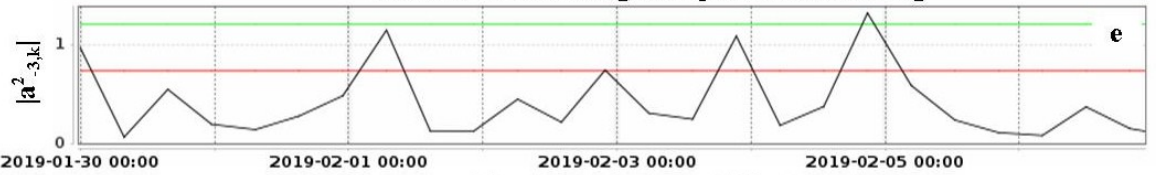

- Errors - $70 \%$ confidence interval - $90 \%$ confidence interval

Fig. 2. Results of foF2 data processing for the period January 30, 2019 - February 6, 2019. 


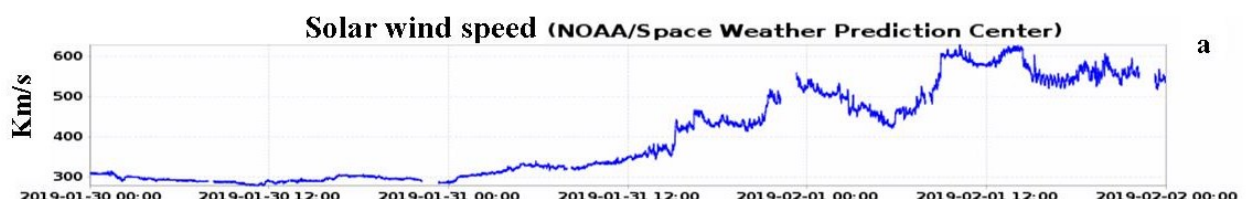

Bz IMF (NOAA/S pace Weather Prediction Center)

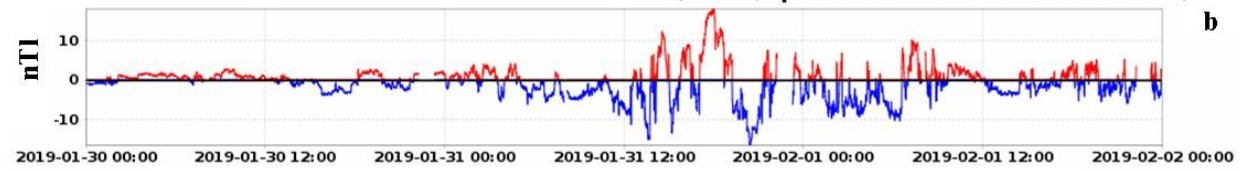

h-component of the magnetic field of the Earth, nTl (Paratunka)

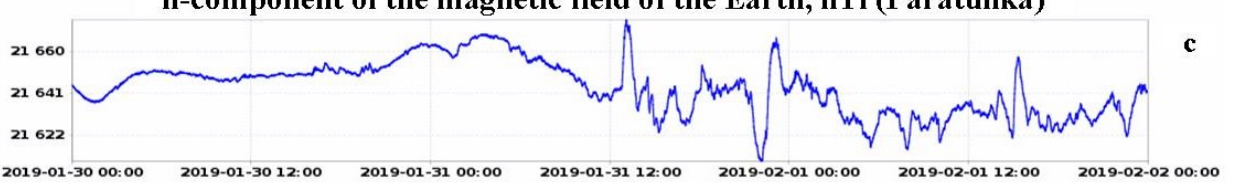

foF2 data

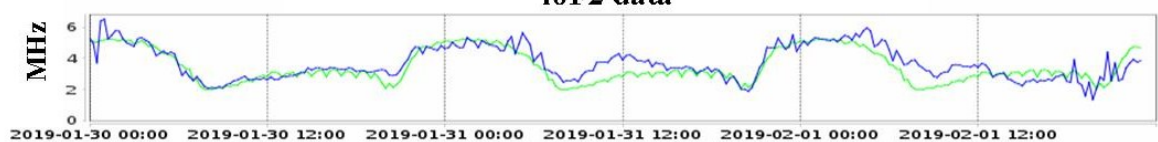

Disturbed periods
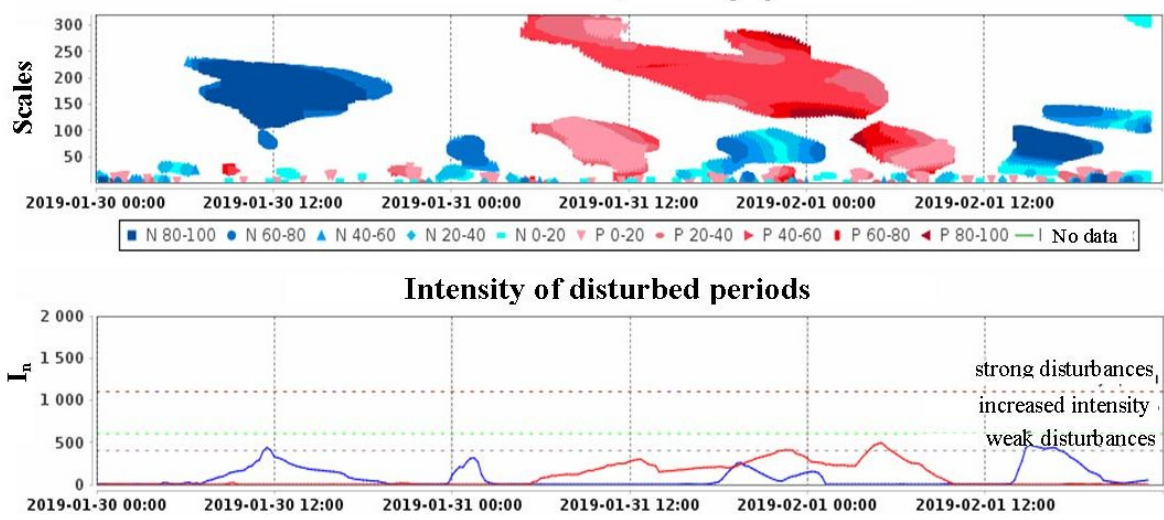
2019.

Fig. 3. Results of foF2 data processing for the period January 30, 2019 - February 1,

Fig. 4, 5 show the results of foF2 ionospheric data processing during the magnetic storm on May 10-11, 2019. According to the data of space weather site (http://ipg.geospace.ru), an accelerated flux from coronal mass ejection (CME from May 6) arrived at the end of the day on May 10, IMF southern component fluctuations increased to $\mathrm{Bz}= \pm 11 \mathrm{nT}$ on May 11 and remained within $\mathrm{Bz}= \pm 6 \mathrm{nT}-\mathrm{Bz}= \pm 8 \mathrm{nT}$ until May 14 (Fig. $5 \mathrm{a}, \mathrm{b}$ ). A positive anomaly of weak intensity with the maximum at about 09.00 (UT) was observed on May 10 (Fig. 5 e, f, operations (6), (7)) before the magnetic storm and several hours before sudden oscillations in the data of solar wind velocity, IMFBz and Earth magnetic field (Fig. 5 a, b, c). Timely detection of positive anomalies preceding magnetic storms and their comparison with space weather data may be used as an additional feature in magnetic and ionospheric disturbance forecast. During the magnetic storm, a multi-scale negative anomaly of high intensity was observed (marked by blue in Fig. 4b, c, operations (6), (7)). At the same time, electron concentration decrease was observed in foF2 data compared to the median values (Fig. 4a). Modeling results (ratio (5), Fig. 4 d,e) show that significant (more than 3 standard 
deviations) and long (more than 2 days) changes (fiductial probability is $70 \%$, Fig. 4 d, e) were observed in ionospheric parameter time variation during electron concentration decrease.

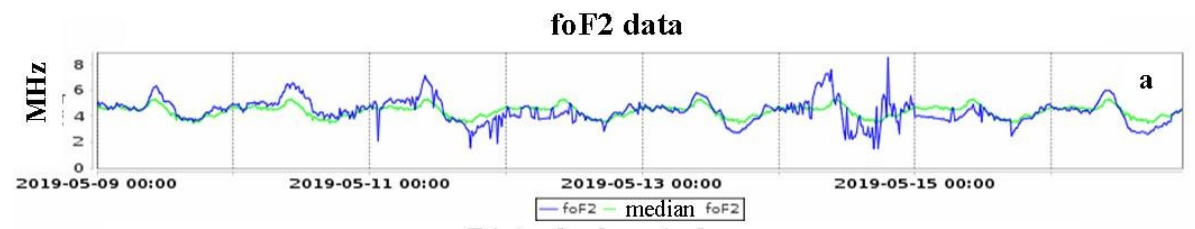

Disturbed periods

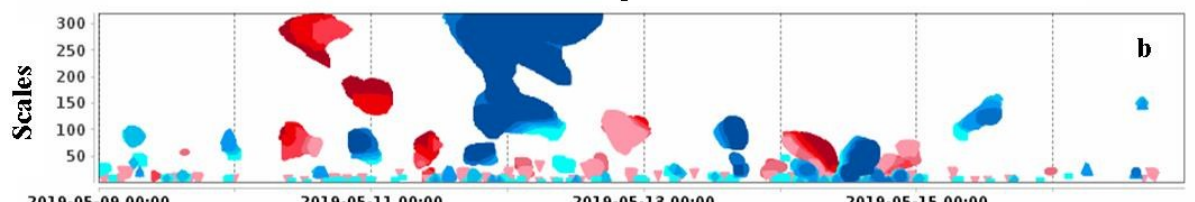

2019.05.09 00:00 2019.05.11 00:00 2019.05.13 00:00

- N 80-100 - N 60-80 \& N 40-60 \& N 20-40 = N 0-20 ₹ P 0-20 - P 20-40 > P 40-60 IP 60-80 \& P 80-100 - No data

Intensity of disturbed periods

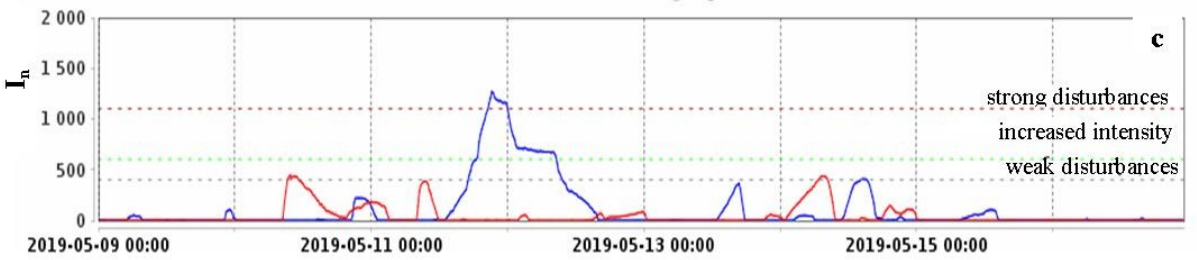

Errors of smoothed component modeling

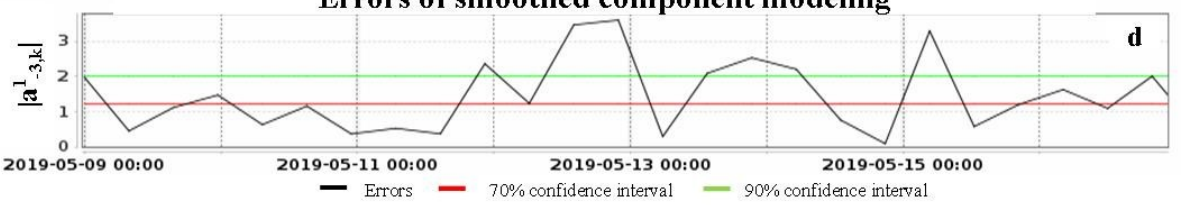

Errors of detailing component modeling

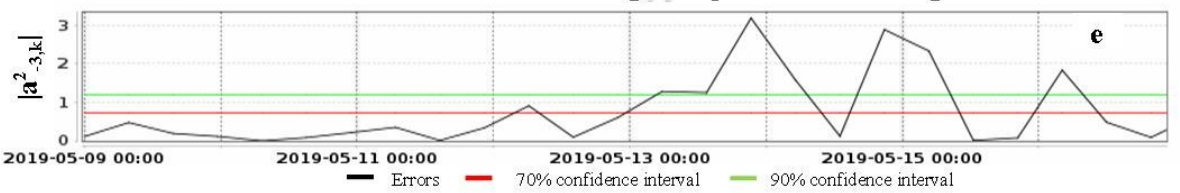

Fig. 4. Results of foF2 data processing for the period May $9-16,2019$. 


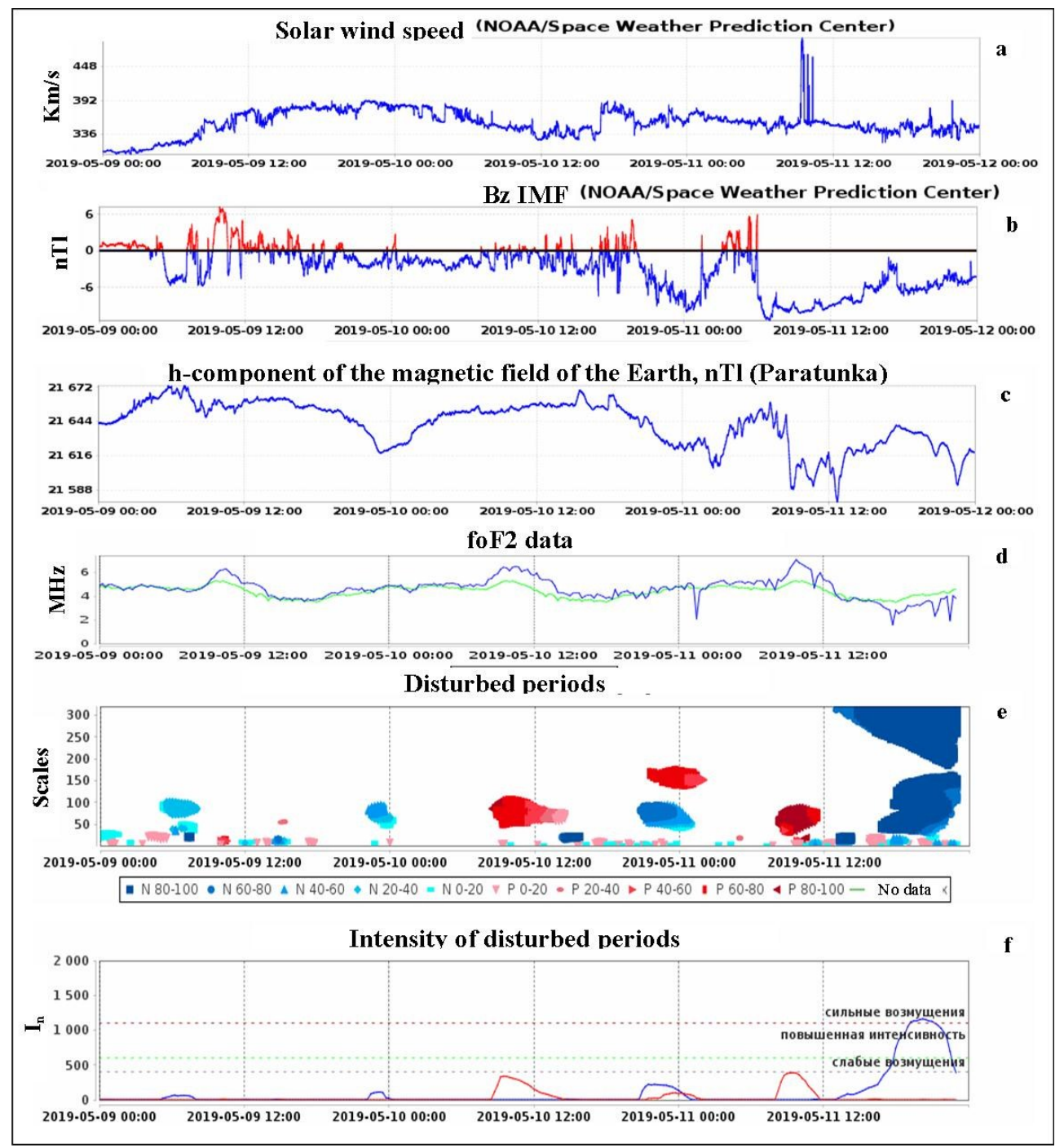

Fig. 5. Results of foF2 data processing for the period May 9-11, 2019.

\section{Conclusions}

The paper shows the results of ionospheric anomalies detection in online mode based on ionosonde data from Paratunka station. Data analysis was carried out by Aurora system for geophysical data online analysis (http://lsaoperanalysis.ikir.ru:9180/1saoperanalysis.html). During the disturbed periods, anomalous changes of different duration and intensity were detected. Anomalous increases of electron concentration in the ionosphere were detected for the events under analysis before magnetic storms. This effect was also observed in the papers [1,2] and may be considered as an additional factor for magnetic storm forecast. Data online analysis gives the opportunity to apply the suggested solutions in space weather forecast tasks. 
The authors appreciate the organizations the geophysical data records of which were applied in this paper.

\section{References}

1. O.V. Mandrikova, N.V. Fetisova, Y.A. Polozov, I.S. Solovev, M.S. Kupriyanov, Earth, Planets and Space, 67 (2015)

2. O. Mandrikova, Yu. Polozov, N. Fetisova, T. Zalyaev, Journal of Atmospheric and Solar-Terrestrial Physics, 181, 116-126 (2018)

3. O. Mandrikova, N. Fetisova, Yu. Polozov, Journal of Physics: Conference Series by IOP Publishing, 1096 (2018)

4. O.V. Mandrikova, N.V. Fetisova, Yu. A. Polozov, V.V. Geppener, Solar-Terrestrial Relations and Physics of Earthquakes Precursors. E3S Web of Conferences, 62, 02003 (2018)

5. A.D. Danilov, Advances in Space Research, 52, 343-366 (2013)

6. J. Lastovicka, Journal of atmospheric and solar-terrestrial physics, 64, 697-605 (2002)

7. M. Nakamura, T. Maruyama, Y. Shidama, Journal of the National Institute of Information and Communications Technology, 56, 391-406 (2009)

8. E.L. Afraimovich, N.P. Perevalova, GPS-monitoring verhnej atmosfery Zemli [GPSmonitoring of the Earth's upper atmosphere] (GU NU RVH VSNC SO RAMN, Irkutsk, 2006)

9. L. Liu, W. Wan, M.L. Zhang, B. Zhao, B. Ning, Journal of geophysical research, 113, 1-12 (2008)

10. G.A. Mansilla, Studia Geophysica et Geodaetica, 51(4), 563-574 (2007)

11. L.F. Chernogor, V.T. Rozumenko, Radio Physics and Radio Astronomy, 13(2), 120137, (2008)

12. A. Mikhailov, B. Morena, G. Miro, D. Marin, Annals of Geophysics, 42(4) (1999)

13. Yu. Polozov, N. Fetisova Solar-Terrestrial Relations and Physics of Earthquakes Precursors. E3S Web of Conferences, 62, 02002 (2018)

14. O.V. Mandrikova, I.S. Solovev, T.L. Zalyaev, Earth Planet Space, 66(1), (2014)

15. O. V. Mandrikova, Yu. A. Polozov, I. S. Solovev, N. V. Fetisova(Glushkova), T. L. Zalyaev, M.S. Kupriyanov, A.V. Dmitriev, Pattern Recognition and Image Analysis, 26, 406-418 (2016)

16. S. Mallat, A wavelet tour of signal processing (Academic Press, London, 1999)

17. G. Box, G. Jenkins, Time series analysis: Forecasting and control (Holden-Day, San Francisco, 1970) 